\title{
Awareness of adverse effects of lead-containing cosmetics among the adolescence and young adult population in Chennai. A survey
}

\author{
Sneha A. ${ }^{1}$, Balaji K. ${ }^{2}$, Yuvaraj Maria Francis ${ }^{3}$, Gunapriya Raghunath ${ }^{4}$ \\ ${ }^{1}$ Undergraduate Students, Saveetha Medical College and Hospital, Thandalam, Chennai, India, ${ }^{2}$ Tutor, Department \\ of Anatomy, Saveetha Medical College and Hospital, Thandalam, Chennai, India, ${ }^{3}$ Assistant Professor, Department \\ of Anatomy, Saveetha Medical College and Hospital, Thandalam, Chennai, India, ${ }^{4}$ Professor \& Head, Department \\ of Anatomy, Saveetha Medical College and Hospital, Thandalam,
}

Chennai, India

\begin{abstract}
Background: Lead and it's compound induced health problems are continue to be an important, preventable public health issue. The common source of lead exposure in modern days is cosmetic products.

Aim and Objectives: The study aims to evaluate the awareness about the adverse effects of lead-containing cosmetics in adolescence and the young adult population including factors influencing customers, knowledge about the product ingredients, and quality.

Materials and Method: In this study, a questionnaire was prepared using Google form and circulated among the adolescence and young adult subjects to assess the awareness about the adverse effect of lead in cosmetics. The study sample comprised of totally 203 participants of both males and females with the age group between 16 to 24 years.

Results: In this study, it is found that $87 \%$ of the participants were aware that cosmetic products harm their health. $78 \%$ of the participants know lead and its compounds present in the cosmetics. $86 \%$ of participants have the habit of checking the ingredients and quality of cosmetic products. About $96 \%$ of the participants were influenced by the advertisement of the product before buying it.
\end{abstract}

Keywords: Awareness, Adolescence, Cosmetics, Lead, and Participants.

\section{Introduction}

Adverse effects of heavy metals are a major public health concern, particularly lead and its compounds may cause negative impacts on human health. Metals such as

\section{Corresponding Author:}

\section{Mr. Balaji Karunakaran}

Tutor, Department of Anatomy, Saveetha Medical College \& Hospital, Saveetha Institute of Medical \& Technical Sciences, Thandalam, Chennai, Tamil Nadu, India 602105

Mobile No.: 9894763124

e-mail:mevsig@gmail.com lead, mercury, and cadmium in cosmetics are described as listed among the ten major chemicals of concern by the World Health Organization (WHO). ${ }^{1}$ These metals are purposefully added to cosmetics, existas impurities in cosmetics, or unknowingly added from manufacturing devices itself. Lead and its compounds occur naturally in the environment. However, most of the lead added throughout the environment by human activities such as car battery, ammunition and as a constituent of solder, alloys, and pewter. The lead in the environment had increased 1,000-fold in the past few centuries especially during the industrial revolution. ${ }^{2}$ The lead has widespread industrial use, such as application in gasoline,fuel and solid waste combustion,paints, and most importantly in cosmetics. Lead compounds 
such as lead dioxide, lead chromate, leadhydroxyl carbonate, and lead sulfide are used in colourcosmetics as pigments. ${ }^{3}$ The risk of exposure to lead is high among children due to their hand to mouth activities which may cause cognitive and neurobehavioral deficits. ${ }^{4}$ The lead toxicities may result in organ damage specifically bone, kidney and liver injuries, intellectual impairment, and other symptoms, such as irritability, headaches, and confusion. ${ }^{5}$ The Food and Drug Administration (FDA) in the United Statesstated that 20 PPM is the maximum limit for lead in edible products. Nevertheless, leadis cumulative, and using lead-contaminated cosmetics regularly, canadd up to significant exposure levels and adversely affect humans. A significant amount of the lead body burden is confined tothe bone with a half-life of more than 20 years (WHO, 1995). ${ }^{6}$ Blood lead level is increased during increased bone turnover especially during pregnancy, lactation, and menopause in women's live. ${ }^{7,8}$ Although several measures to control the lead exposure are already implemented by both government agencies and non-governmental organizations in many countries such as the use of unleaded gasoline, unleaded paint, ceramics used for storage and preparation of food and lead-free cosmetics. Lead contamination is still a major public health problem in underdeveloped and developing countries and targeted high-risk populations. Lead compounds and several other substances are prohibited ingredients in cosmetics in many countries. ${ }^{9}$ Therefore, the aim of the study is to understand the level of awareness and knowledge about lead-containing cosmetics and its adverse effects among its users.

\section{Methodology}

This cross sectional study was conducted in Saveetha Medical College during the period from November 2019 to April 2020. This cross-sectional study was to assess the awareness about the composition of cosmetics particularly about the potentially harmful substances such as lead. A survey method was adopted as a means of data collection because it provided the best possible way to assess the awareness of participants regarding the use of cosmetics. This study was conducted by using Google form to observe the cosmetic usage practices of participants through the medium of analyzing questionnaires. The survey was arranged in three sections in which the first section consisted of questions regarding the age and usage of makeup. The second section consisted of questions regarding the usage pattern of different personal care products. The third section of questions included questions regarding the awareness and shopping patterns of the consumers. The data was collected from a sample size of 203 participants including both the gender within the age group of 16 to 24 years with proper informed consent. The data collected were compiled and analyzed using Microsoft excel 2010.

\section{Results}

The mean age group of the study participants was 19.1 years with a standard deviation of 1.347. The majority of the participants were females $157(77 \%)$ and males were $46(23 \%)$.

The majority of the participants were aware of the fact that cosmetic products have harmful compounds (87\%) (Fig: 1).

The eye-opener finding was that $78 \%$ of the participants have knowledge about the adverse effects of lead and its compounds present in the cosmetics (Fig: 2).

About $6 \%$ of participants did not state the frequency of cosmetic use. Of the remainder $67 \%$ reported having used cosmetics rarely, $21 \%$ used cosmetics frequently, and $6 \%$ very frequently in their life. Figure: 3 shows the frequency of the use of cosmetics by the participants. Respondents were asked if they had ever used cosmetics for the whole day, and if so how many cosmetics they used simultaneously. This question was not answered by the majority of the participants, and $56 \%$ stated that they had never been daily users. Above $27 \%$ of the participants who had been daily users at some time, the mean number of cosmetics used per day was 4 (range 1- 7).

The majority of participants $86 \%$ check the ingredients and quality of the cosmetic products, meanwhile, $11 \%$ and $3 \%$ of the participants just see the price and package respectively without seeing the quality and ingredients of the products (Fig: 4). And about 96\% of the participants were influenced by the advertisement of the product before buying it.

\section{Discussion}

The important reason for carrying out this crosssectional study is to understand the awareness and knowledge about the adverse effects of lead-containing cosmetics. The purpose for selecting lead and its compound content in cosmetics for this study was to add weight age to the recent reports about the presence of lead in cosmetic products such as lipsticks and hair 
dyes and also since the potential exposure to lead may be harmful even with ordinary use. Moreover, lead proven to cause serious health problems, including poisoning, and pathological change in vital organs. ${ }^{10,11}$ The study done by Sharafi $\mathrm{K}$ et al stated that lead content in six tested lipstick samples was evaluated; the highest and lowest lead content was $455 \mathrm{mg} / \mathrm{kg}$ and $208 \mathrm{mg} / \mathrm{kg}$ respectively. This difference of lead content may be attributed to the difference in the quality of the raw materials used in the lipsticks. $^{12}$

This survey has questions that are basics one to tests the awareness of the participants about the leadcontaining cosmetics including questions such as usage, knowledge of quality and ingredients, awareness about adverse health effects, and preventive measures. Similarly, study was conducted by Shiraz A, and Rahaman A reported that $88.33 \%$ of respondents used cosmetic products only once a day. $77.33 \%$ of respondents spent more than Rs. 1000 per year on their expenditure on cosmetics, and the same percentage of respondents were found using multiple cosmetic items in a day. ${ }^{13}$ In 2016, study done among medical students from Kerala stated that $83.75 \%$ males and $98.3 \%$ females use cosmetics, Among them, $80 \%$ preferred natural cosmetics and respondents were influenced by beauty experts (6.7\%); by advertisement(12\%); dermatologists $(33.56 \%)$ and by the peer group (33.56\%). ${ }^{14}$
A predominant number of women $(84.2 \%)$ in Bolgatanga, Ghana were not aware of the adverse effects of cosmetic bleaching products. However, $60.8 \%$ do not bleach their skin and $39.2 \%$ used cosmetic bleaching products. $^{15}$

The continuous use of cosmetic products contaminated with lead among the young population who already have adequate knowledge about the adverse effects of cosmetics is a major concern where extensive use of lead-containing cosmetics should be avoided until it is adequately addressed. Therefore, efforts must be made to inform the users and the general public especially the young population, pregnant and lactating women and children about the harmful consequences of cosmetic use.

In comparison with lead content from sources such as food, water, and air, continuous exposure to lead from cosmetics has been considered as a negligible source. Despite that, consistent and cumulative exposure of lead in the body for a long time, cosmetics can be considered as a substantial source of the lead. ${ }^{16,17}$ Therefore, to cancel the adverse health effects of lead, cosmetics producers must use natural ingredients specifically colour additives that are lead-free.

\section{Knowledge about adverse effects of Cosmetics}

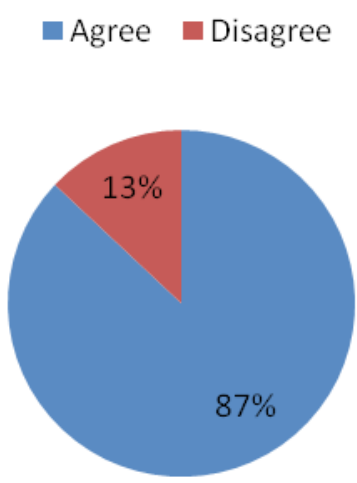

Figure 1: Showing knowledge about the adverse effects of Cosmetics 


\title{
Awareness about the presences of Lead in Cosmetics
}

$$
=\text { Yes } m \text { No }
$$

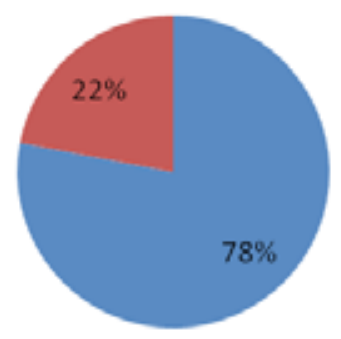

Figure 2: Showing knowledge presence of lead and its compounds in the cosmetics

\section{Frequency of using cosmetics}

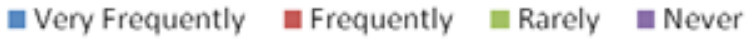

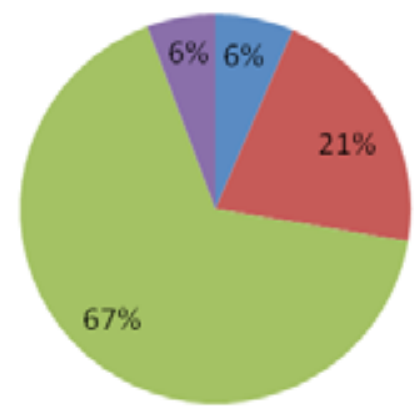

Figure 3: Showing the frequency of cosmetic use among the participants

\section{Factors influencing Cosmetic purchasing decision}

$$
\text { =Quality = Ingredients = Price = Packaging }
$$

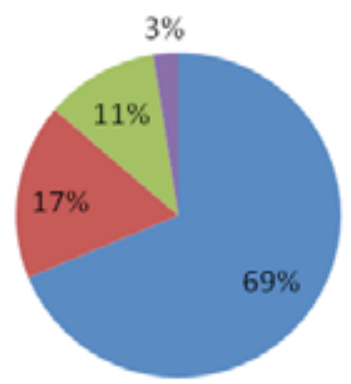

Figure 4: Showing the factors influencing the cosmetic purchasing decision 


\section{Conclusion}

The data revealed useful information about knowledge of lead content in cosmetic products among the adolescences from Chennai. Overall, the study provided a basic understanding of the participant's attitude towards cosmetic products. Public health interventions should focus on prevention to ensure that lead contenting cosmetics are not available for sale in the market or through e-commerce. The elimination of lead-containing cosmetics from stores, checking the ingredients to determine its lead level, and conducting the routinetest for the safety products are the other preventive measures. Health professionals can play a major role in raising awareness through education to reduce risk.

Acknowledgments: The authors are thankful to all the faculties of the Department of Anatomy, Saveetha Medical College and Hospital, Saveetha Institute of Medical And Technical Sciences (SIMATS), Chennai, India, for the support and gratitude to carry out this work.

Authors Contribution: Sneha A made a major contribution to data collection, experimental work. Balaji Karunakaran has made involvement in experimental work and drafting the manuscript, Yuvaraj Maria Francis has made significant involvement in the interpretation of data. Gunapriya Raghunath participated in the design of the study and coordination to draft the manuscript.

\section{Funding: Self-funding}

Conflicts of Interest: The authors don't have any conflicting interests.

Ethical Approval: This study does not involve any human or animal testing.

\section{References}

1. Preventing Disease through Healthy Environments, Action is needed on chemicals of major public health concern. World Health Organization, Geneva, Switzerland, 2010 https://www.who.int/ ipcs/features/10chemicals_en.pdf

2. Pinho $\mathrm{S}$ andLadeiro B. Phytotoxicity by Lead as Heavy Metal Focus on Oxidative Stress. Journal of Botany, vol. 2012, Article ID 369572, 10 pages.

3. Borowska S, Brzoska MM. Metals in cosmetics: implications for human health. J ApplToxicol. 2015,35:551-72.
4. Agency for Toxic Substances and Disease Registry. Toxicological profile for lead. Atlanta, GA: US Department of Health and Human Services, Agency for Toxic and Disease Registry; 2007.

5. Gidlow DA. Lead toxicity. Occup Med. 2015,65:348-56.

6. WHO, Environmental Health Criteria 165: Inorganic Lead, International Programme on Chemical Safety. World Health Organization, Geneva, Switzerland, 1995.

7. Gulson BL, Jameson CW, Mahaffey KR, Mizon KJ, Patison N, Law A. Relationship of lead in breast milk to lead in blood, urine, and diet of the infant and mother. Environ Health Perspect, 1998, 106:667-674.

8. Ettinger AS, Tellez-Rojo MM, Amarasiriwardena C, Peterson KE, Schwartz J, Aro A. Influence of maternal bone lead burden and calcium intake on levels of lead in breast milk over the course of lactation. Am J Epidemiol2006, 163: 48 -56.

9. Bocca B, Pino A, Alimonti A, Forte G. Toxic metals contained in cosmetics: A status report. RegulToxicol Pharmacol 2014,68:447-67

10. Shrivas, K. and Patel, D.K. Separation and preconcentration of trace level of lead in one drop of the blood sample by using graphite furnace atomic absorption spectrometry. J. Hazard. Mater. 2010, 176, 414-417.

11. Koller, K., Brown, T., Spurgeon, A. and Levy, L. Recent developments in low-level lead exposure and intellectual impairment in children. Environ. Health Perspect. 2004, 112, 987-994.

12. Sharafi K, Fattahi N, Pirsaheb M, Yarmohamadi M and FazlzadehDavil M. Trace determination of lead in lipsticks and hair dyes using microwave-assisted dispersive liquid-liquid microextraction and graphite furnace atomic absorption spectrometry. International Journal of Cosmetic Science, 2015, 37, 489-495.

13. Shiraz A, Rahaman A. Study on Awareness about Adverse Health Effects of Cosmetics among Females of Different Age Groups. JMSCR, 2019,07; 11; 503-510.

14. Samson JF, Philip M, Arjun C, Varadan S. Trends in awareness and use of cosmetics among medical students - a study from a medical college in South Kerala, India. International Journal of Contemporary Medical Research 2017;4 (6):1415- 
1417.

15. Richard A. Kuffour, Dartey E, Owusu W and Philip M. DabuohLevel of Awareness of Effects of the Use of Cosmetic Bleaching Products among Women: A Case Study of Bolgatanga Municipality of Ghana. Research on Humanities and Social Sciences, 2014, $4 ; 11$.
16. Nnorom I C, Igwe $\mathrm{J} \mathrm{C}$, and Oji-Nnorom $\mathrm{C} \mathrm{G}$, Tracemetal contents of facial (make-up) cosmetics commonly used in Nigeria. African Journal of Biotechnology, 2005, 4; 10 1133-1138.

17. Castro-Gonz'alezM Iand 'endez-Armenta M M. Heavy metals: implications associated to fish consumption.Environmental Toxicology and Pharmacology, 2008, 26; 3; 263-271. 